\title{
Crucial role of decoherence for electronic transport in molecular wires: Polyaniline as a case study.
}

\author{
Carlos J. Cattena, ${ }^{1}$ Raúl A. Bustos-Marún, ${ }^{1,2}$ and Horacio M. Pastawski ${ }^{1}$ \\ ${ }^{1}$ Instituto de Física Enrique Gaviola (CONICET-U.N.C.) and Facultad de Matemática Astronomía y Física, \\ Universidad Nacional de Córdoba, Ciudad Universitaria, Córdoba, 5000, Argentina and \\ ${ }^{2}$ Facultad de Ciencias Químicas, Universidad Nacional de Córdoba, \\ Ciudad Universitaria, Córdoba, 5000, Argentina.
}

\begin{abstract}
In this work we attempt to elucidate the nature of conductivity in polymers by taking the acidbase doped polyaniline (PAni) polymer. We evaluate the PAni conductance by using realistic $a b$ initio parameters and including decoherent processes within the minimal parametrization model of D'Amato-Pastawski. In contrast to general wisdom, which associates the conducting state with coherent propagation in a periodic polaronic lattice, we show that decoherence can account for high conductance in the strongly disordered bipolaronic lattice. Hence, according to our results, there is no need of considering a mix model of "conducting" polaronic lattice islands separated by "insulating" bipolaronic lattice strands as is usually assumed for PAni. We find that without dephasing events, even very short strands of bipolaronic lattices are not able to sustain electronic transport. We also include a discussion of specific mechanisms that should be involved in decoherence rates of PAni and relate them with Marcus-Hush theory of electron transfer.
\end{abstract}

PACS numbers: 72.80.Le, 72.10.-d, 73.20.Jc, 72.15.Rn

\section{INTRODUCTION}

In the late 70's, Alan MacDiarmid, Alan Heeger and Hideki Shirakawa led the investigations which put conducting polymers at the center stage by unraveling the transition from insulator to metal upon doping of polyacetilene [1]. The following decades, these materials encountered numerous technological applications [2, 3]. The novelty of polyacetilene's physical properties, e.g. transport through solitonic excitations [4], made it the most intensively studied conductive polymer. However, the interest then shifted to polyanilines [5] and related compounds because they are inexpensive, stable and easy to made.

In spite of its long history, polyaniline (PAni) became a new paradigm for polymeric conductors as it shows a dramatic increase in conductivity either by acidic treatment or by electrochemical oxidation. In spite of this fact, the physical basis of its transport mechanism and of the insulator-metal transition proved more elusive. Starting from a semiconducting PAni in an emeraldine base form (Fig. 1-a), protonation leads to an internal redox reaction that converts it into a metal (emeraldine salt). In order to account for the highly conducting nature of this doped polymer there are two well established models that imply two different lattice arrangements. These are associated to the appearance of two possible charged defects upon protonation. On one hand, the polaronic lattice (PL), which describes a lattice of Nitrogen bridged benzene rings that becomes fully periodic in the case of $100 \%$ of protonation. Even when one of every two Nitrogens is in the form $\mathrm{N}^{+}$supporting a polaron, the corresponding $\mathrm{p}_{z}$ electrons form a collective band of Bloch extended states which, being half-filled, behaves as a metal (Fig. 1. c). On the other hand, in a crystalline bipolaronic lattice (BL) the protonated quinoid units $\left(\mathrm{NH}^{+}=\mathrm{Q}=\mathrm{NH}^{+}\right)$ are bridged by three benzene rings. The electron tunneling between neighbor $\mathrm{NH}^{+}$units, leads to a bonding basic unit that justifies a bipolaronic description. Natural disorder appears through the fluctuation of the bridge length (Fig. 1 1 b). Hence, while further tunneling between units could be possible, within the standard wisdom, disorder ensures localized eigenstates that prevent propagation [6]. Galvão et al. [7] concluded that disorder should be an essential ingredient in these systems. They made molecular orbitals calculations of the electronic structure of PAni chains which showed that disorder pulls the Fermi energy down through the localized states of the valence band. Later on, $\mathrm{Wu}$ and Phillips 8] agreed with Galvão in the role of the protonation, further showing that induced disorder can be identified with a Random Dimer Model (RDM) [9, 10]. By adopting Landauer's view that "conductance is transmission" [1], the current motto of molecular electronics [12], it was proved that the short range order of the RDM produces a set of delocalized or propagating states [9, 10, 13]. This opened the possibility that Fermi energy might lie in a delocalized region. However, Farchioni et al. [14], by using an $a b$ initio parametrization, made a detailed tightbinding based study of PAni-HCl comparing the $\mathrm{BL}$ and PL models [15]. They showed that even when the BL model exhibits extended states, its Fermi energy is far from the high transmission regions. These ideas seemed to support the PL as the only PAni emeraldine salt capable of metallic behavior. Indeed, the observation of Pauli susceptibility on conducting samples was attributed to extended states in a polaronic lattice [16]. However, firstprinciple energy stability calculations point into the opposite direction. A BL is by far the more stable energy configuration when compared to a PL [17] or its variants [18]. A picture that could unify these conclusions is that of segregated metallic (PL) regions and insulating 
(BL) domains. Transport would be mediated by hopping between metallic fibers in the polymer backbone [19]. However, it is not clear that such structures could give a lower free energy than a pure BL one. Besides, in this model disordered islands would constitute the conductance bottle-neck for which a microscopic description is lacking. Further emphasizing the role of BL, it was recently suggested that susceptibility experiments could not be used to rule out the bipolaronic structure from conducting samples. This is because an internal chemical redox equilibrium between bipolaronic structures and a number of polaronic defects with Curie susceptibility, should manifest as an overall susceptibility whose temperature dependence would be indistinguishable from the Pauli paramagnetism [20]. In summary, the early works associate the conducting state of PAni with periodic order because the existence of extended Bloch eigenstates is a condition for coherent propagation.

In order to account for the surprising frequency dependence of the dielectric constant and of the conductivity observed on conjugated polymers [21], Prigodin and Epstein [22] suggested a new mechanism of charge transport. They argued that the metallic state of polymers like PAni is sustained by a granular picture of transport where metallic islands, separated by amorphous material, interact through intrachain resonant tunneling events in a quasi 1-D variable range hopping theory. However, after an energy scale analysis, Martens et al. [23, 24] arrived to the conclusion that intrachain charge carrier delocalization should extend over several grains. In consequence, there is some critical mechanism that governs the formation of truly delocalized states. They propose a quasi 1-D model of weakly coupled disordered chains with phase-breaking events that are modeled in the Landauer-Büttiker framework. In this case the 1-D Schrödinger wave function picture for a single chain remains essentially correct with the additions of a finite lifetime, i.e. decoherence, due to dephasing events. Their source can be multiple, ranging from electron-phonon coupling [25, 26] to through-space electron-electron interactions between charge fluctuations [27], or even a weak interchain coupling [28]. Increasing the interchain coupling eventually will give rise to a transition from a quasi 1 -D to a fully 3 -D behavior as demonstrated by numerical simulations [29, 30]. However, for conjugated polymers such as PAni, the interchain charge transfer is weak and a 1-D model that includes decoherence should be a good approximation. Within this framework, Martens et al. invoke dimensional arguments that explain the anomalous frequency dependence of the dielectric constant and conductivity of several polymers. However, their conclusions are based on estimations of the relevant system quantities.

In this work we attempt to elucidate in detail the nature of conductivity of polymers by taking the PAni bipolaron lattice structure as the case study. For that purpose, we use realistic $a b$ initio based tight-binding parameters which can be easily reduced [31] to the min-

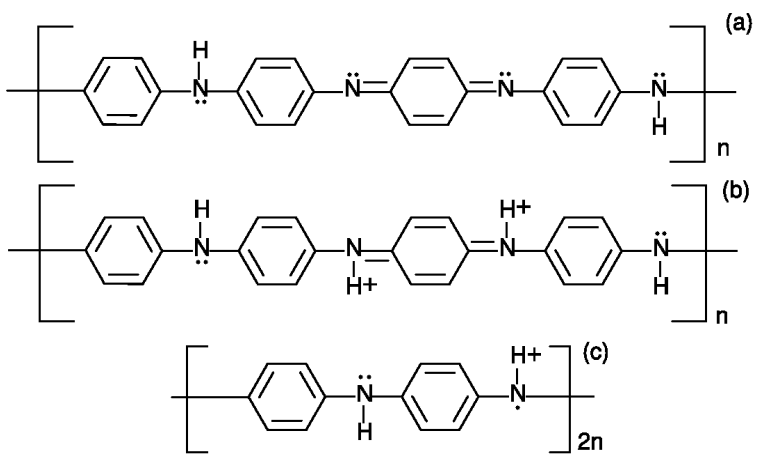

FIG. 1. Three different forms of PAni: (a) Emeraldine base, and two lattice models after a doping process, (b) Bipolaron, (c) Polaron

imal parametrization of the D'Amato-Pastawski (DP) model [32]. This provides a simple solution to the otherwise complex Keldysh formulation of transport [33, 40]. Indeed, this strategy was applied before to PAni by Maschke et al. and Schreiber et al. [34 36]. However, they focused on already conducting PAni polaronic chains that are affected by decoherence and/or interchain coupling. This also sums to the recent efforts in including decoherent processes in molecular electronics [37 39]. At this point a comment on the meaning of decoherence seems in order since nowadays this term is mostly used in connection with quantum information theory, where it denotes the (full or partial) collapse of a pure quantum state due to usually unspecified interactions with the environment. This might seem different from the usual language of electronic transport in the solid state. There, one is used to deal with specific interactions, such as those with phonons, magnetic impurities or other electrons, which may involve transitions with given selection rules, e.g. $|k\rangle \rightarrow|k+q\rangle$. These transition probabilities are evaluated within a Fermi Golden Rule (FGR). In this approximation, the coupling with the extra degrees of freedom, the environment, prevents the interference among the component remaining in $|k\rangle$ with that in $|k+q\rangle$. Thus, with some probability the environment "measures" an electron in the state $|k\rangle$ and "re-injects" it incoherently in $|k+q\rangle$. These processes, which usually involve some inelasticity, are inherently different from the elastic scattering with imperfections and impurities that produce the interferences leading to localization [ [6] Quite often one realizes that, regardless of the specific selection rule, the relevant role of interactions is just to provide for decoherence, a mechanism that competes with the coherent scattering that results in localization. This is precisely the spirit of the "local phonon" bath or the fictitious voltage probes that lead to the imaginary site energies introduced in the DP model [33, 40]. Thus, the idea is that decoherence from the system-environment interaction provides a knob that sweeps transport between a Mott's variable range hopping regime and a typical 1- 
D metal [40, 41]. Transport in conducting PAni should occur somewhere at this crossover.

We start in section II by recalling the Landauer formulation for the conductance and introducing the DP model for decoherent transport. In Section III we describe the application of this model to the BL structure of PAni and we show in Section IV the effective transmission results and associated currents in the non-linear regime. In section $\mathrm{V}$ we present the main conclusion: at room temperature decoherence can account for high conductance in the strongly disordered bipolaronic lattice. The mechanisms that can contribute to decoherence are discussed in an Appendix. There, we asses the possible role of interchain coupling and various forms of electronphonon interactions. Moreover, a detailed treatment of these specific models allows us to enlight their differences and similarities with the well-known Marcus-Hush theory [42] for vibration-assisted electron transfer.

\section{EFFECT OF DECOHERENCE IN THE CONDUCTANCE}

The Landauer formulation requires to calculate the transmission of the system. Assuming that the sample's Hamiltonian is known one must incorporate explicitly the leads connecting to the electrodes. Besides, to take account of decoherent interactions with an environment DP included several phase-breaking fictitious probes [43]. Thus, an original molecular orbital Hamiltonian with $N$ orbitals,

$$
\hat{H}_{S}=\sum_{i=1}^{N}\left(E_{i} \hat{c}_{i}^{+} \hat{c}_{i}+\sum_{j>i}^{N}\left[V_{i, j} \hat{c}_{i}^{+} \hat{c}_{j}+V_{j, i} \hat{c}_{j}^{+} \hat{c}_{i}\right]\right),
$$

becomes an effective Hamiltonian that incorporates the leads and the interactions with the environment:

$$
\hat{H}_{\text {eff. }}=\left(\hat{H}_{S}-\mathrm{i} \eta \hat{I}\right)+\hat{\Sigma}_{L}+\hat{\Sigma}_{R}+\hat{\Sigma}_{\phi},
$$

where $\hat{\Sigma}_{L}=\Sigma_{L} \hat{c}_{1}^{+} \hat{c}_{1}$ and $\hat{\Sigma}_{R}=\Sigma_{R} \hat{c}_{N}^{+} \hat{c}_{N}$ are, respectively, the self-energies operators describing the escape to the left and the right leads obtained through a Dyson equation,

$$
\begin{aligned}
\Sigma_{L(R)} & =\frac{V^{2}}{\varepsilon-\left(E_{0}-\mathrm{i} \eta\right)-\Sigma_{L(R)}} \\
& =\Delta_{L(R)}(\varepsilon)-\mathrm{i} \Gamma_{L(R)}(\varepsilon),
\end{aligned}
$$

where $\Gamma_{L(R)}$ results proportional to the escape rate, and hence to the Fermi velocity, at the $L(R)$ lead. We include the effects of the incoherent processes in the Hamiltonian, e.g. electron-phonon or through space electron-electron, simply through an imaginary correction to selected site eigenenergies

$$
\hat{\Sigma}_{\phi}=\sum_{l}-\mathrm{i} \Gamma_{\phi} \hat{c}_{l}^{+} \hat{c}_{l}
$$

Hence, $\Gamma_{\phi}$ is an energy uncertainty associated to a decay rate of the local state at site $l$ described by the FGR. We drop any possible dependence on $l$ simplifying the description. Since $\hat{I}$ is the identity operator, $\eta$ can be taken as an infinitesimal imaginary part of the local energy, $E_{i} \rightarrow E_{i}-$ i $\eta$, resulting in a decay to the environment in the same sense as the $\Gamma$ 's above.

Given the effective Hamiltonian we have the retarded and advanced Green's functions in terms of the real en$\operatorname{ergy}$ variable $\varepsilon$ :

$$
\begin{aligned}
\mathbb{G}^{R}(\varepsilon) & =\left[\varepsilon \mathbb{I}-\mathbb{H}_{\text {eff. }}\right]^{-1} \\
& =\mathbb{G}^{A \dagger}(\varepsilon),
\end{aligned}
$$

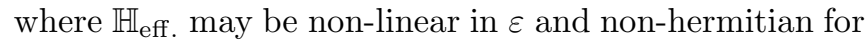
$\eta \neq 0$. The imaginary part of the Green's function enable to evaluate the local density of states at site $i$ as:

$$
N_{i}(\varepsilon)=-\frac{1}{\pi} \lim _{\eta \rightarrow 0^{+}} \operatorname{Im} G_{i, i}^{R}(\varepsilon) .
$$

In a closed system, this is also obtained diagonalizing the Hamiltonian.

According to the optical theorem, the local density of states,

$$
\begin{aligned}
N_{j}(\varepsilon) & =\frac{1}{\pi \sum_{\beta=L, R, \phi} \Gamma_{\beta j}(\varepsilon)} \times \\
& \sum_{\substack{i=1 \\
\text { sites } \alpha, \beta=L, R, \phi}}^{N} \sum_{\beta j}(\varepsilon) G_{j, i}^{R}(\varepsilon) \Gamma_{\alpha i}(\varepsilon) G_{i, j}^{A}(\varepsilon),
\end{aligned}
$$

can be cast as a flux between the asymptotic states described by a generalized Fisher-Lee formula. We adopt the notation $\Gamma_{L} \equiv \Gamma_{L 1}, \Gamma_{R} \equiv \Gamma_{R N}, \Gamma_{\phi} \equiv \Gamma_{\phi i}$, to emphasize that each site can decay through different processes, e.g. $\alpha, \beta \in\{L, R, \phi\}$ are the possible decay processes taking place at sites $i, j \in\{1, \ldots, N\}$. With this notation the generalized transmission probability results:

$$
T_{\alpha i, \beta j}(\varepsilon)=2 \Gamma_{\beta j}(\varepsilon) G_{j, i}^{R}(\varepsilon) 2 \Gamma_{\alpha i}(\varepsilon) G_{i, j}^{A}(\varepsilon) .
$$

Within the DP model current conservation is imposed at each site and energy $\varepsilon$. This requires an incoherent re-injection of every electron that has decayed through the $\Gamma_{\phi i}$. This leads to the evaluation of the kernel $\mathbb{W}^{-1}$, describing incoherent density propagation, where [43]:

$$
W_{i, j}=\left[\left(\sum_{k=1}^{N} T_{\phi k, \phi i}\right) \delta_{i, j}+T_{\phi i, \phi j}\left(1-\delta_{i, j}\right)\right] .
$$

From this we obtain the effective transmission through the sample:

$$
\widetilde{T}_{R L}=T_{R N, L 1}+\sum_{i, j=1}^{N} T_{R N, \phi i}\left[\mathbb{W}^{-1}\right]_{i, j} T_{\phi j, L 1} .
$$

The right hand side of Eq. 12 contains two contributions: the first one represents electrons that propagate 


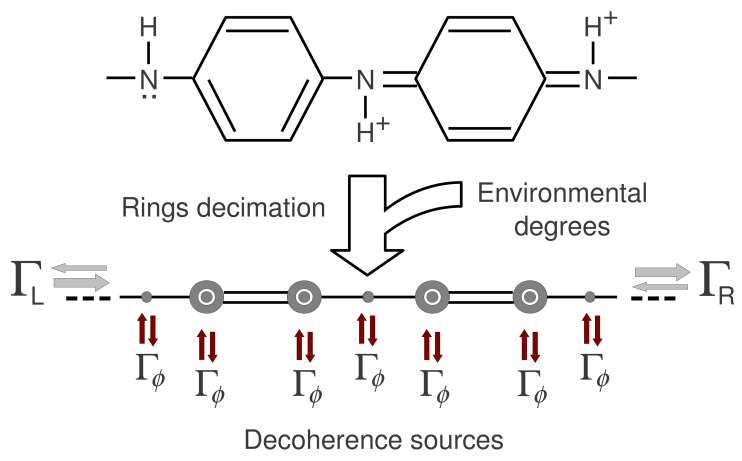

FIG. 2. Diagrammatic representation of benzenoid rings decimated to obtain equivalent renormalized units in one dimension. Incoherent channels of D'Amato-Pastawski model also shown.

coherently through the sample, the second term contains the incoherent contributions due to electrons that suffer their first collision at site $i$ and their last collision at site $j$.

Since, $\widetilde{T}(\varepsilon)$ piles up all vertical processes into the energy $\varepsilon$, one can finally approximate the net current through the sample by:

$$
\begin{aligned}
\mathrm{I} & =\frac{2 e}{h} \int \widetilde{T}_{R L}(\varepsilon)\left[f_{L}(\varepsilon)-f_{R}(\varepsilon)\right] \mathrm{d} \varepsilon \\
& \simeq \frac{2 e^{2}}{h} \widetilde{T}_{R L}\left(\varepsilon_{F}\right) \mathrm{V} \equiv \mathrm{GV},
\end{aligned}
$$

where $f_{R(L)}$ is the Fermi distribution function at the right (left) lead and the factor 2 accounts for spin degeneracy. Here, we assumed that an electron with energy $\varepsilon$ coming from the left lead arrives to the right lead with the same energy, which is only true for very small $\Gamma_{\phi}$. The second line is the linear approximation for infinitesimal voltage $\mathrm{V}$ and temperature, where $\mathrm{G}$ accounts for conductance.

\section{THE PANI MODEL}

We consider a fully protonated BL, which we expect to correspond to the highly conducting emeraldine salt. By decimation of the benzenoid rings as it is shown in figure 2, we reduce the PAni emeraldine salt chain to onedimensional effective system [31]. Each ring is replaced by the proper renormalized sites at the place of the paraCarbon atoms.

The sample Hamiltonian results:

$$
\hat{H}_{S}=\sum_{j=1}^{N}\left(E_{j} \hat{c}_{j}^{+} \hat{c}_{j}+V_{j} \hat{c}_{j}^{+} \hat{c}_{j+1}+V_{j} \hat{c}_{j+1}^{+} \hat{c}_{j}\right) .
$$

When $j=3 s+1$ with $s$ positive integer, $E_{j}$ is the Nitrogen $\mathrm{p}_{z}$-orbital energy and $V_{j}$ is the $\pi$ binding energy (hopping) between the nitrogen and the para- $\mathrm{C} \mathrm{p}_{z}$-orbitals.
When $j=3 s$ and $j=3 s-1$ we have the renormalized parameters for para-C $\mathrm{p}_{z}$-orbitals:

$$
V_{j}=\frac{V_{o o} V_{p o}^{2}}{\left(\varepsilon-E_{o}\right)\left(\varepsilon-E_{o}-\frac{V_{o o}^{2}}{\varepsilon-E_{o}}\right)},
$$

and

$$
E_{j}=E_{p}+\frac{V_{p o}^{2}}{\varepsilon-E_{o}-\frac{V_{o o}^{2}}{\varepsilon-E_{o}}},
$$

where $E_{o}$ and $E_{p}$ are bare site energies for electrons in the $\mathrm{p}_{z}$-orbitals of ortho- $\mathrm{C}$ and para- $\mathrm{C}$ respectively; $V_{o o}$ is the hopping between ortho- $\mathrm{C}$ and $V_{p o}$ is the hopping between a para-C and ortho-C. In this work we use the tight binding parametrization of Vignolo et al. [15] for the bipolaron lattice model of base-emeraldine doped with $\mathrm{HCl}$.

We will consider decoherent sources on effective electron $\mathrm{p}_{z}$-orbitals sites by including a constant imaginary correction to the site energy as in Eq. 5. This is the most convenient choice for computational purposes. A first principles calculation of this imaginary correction is beyond the scope of this work. Its complexity lying on the multiple effects that must be considered. In the appendix we discuss in certain detail two mechanisms: interchain tunneling and the effect of torsional modes on the crucial $\pi$ bonds. However, it is enough to resort to dimensional arguments based on spontaneous symmetry breaking of the quantum coherent state 44]. The reasoning takes into account that quantum bosonic modes with energies $\leq k_{B} T$ should be occupied by many quanta, indicating that $\Gamma_{\phi}$ should be of the order of $k_{B} T$ [24]. In accordance with this general framework, Rebentrost et al. obtain a comparable estimation for $\Gamma_{\phi}$ for excitons in photosynthetic complexes interacting with a phonon bath [45]. Indeed, experiments on DNA strands fits $\Gamma_{\phi}$ of this order using the DP model 46]. Thus, we fix an effective $\tilde{\Gamma}_{\phi}$ on each effective site such that the energy uncertainty per orbital site is $\Gamma_{\phi}=k_{B} T$. We will see in the next section that small variations of the precise value of $\Gamma_{\phi}$ have little impact on conductance.

Right and left leads are described by Eq. 3 choosing $E_{0}=0$ and $V=5 \mathrm{eV}$ to observe the appropriate bandwidth of interest $[-10 \mathrm{eV}, 10 \mathrm{eV}]$. Furthermore, we calculated the Fermi energy by diagonalizing the exact tightbinding Hamiltonian for different configurations. For all possible chain arrangements, the Fermi level is nearly the same, around the average.

\section{NUMERICAL RESULTS}

We have performed a detailed analysis of the conductance properties of the BL model of polyaniline emeraldine salt. Due to the fact that, according to experimental data, PAni chains seem to have an average length 

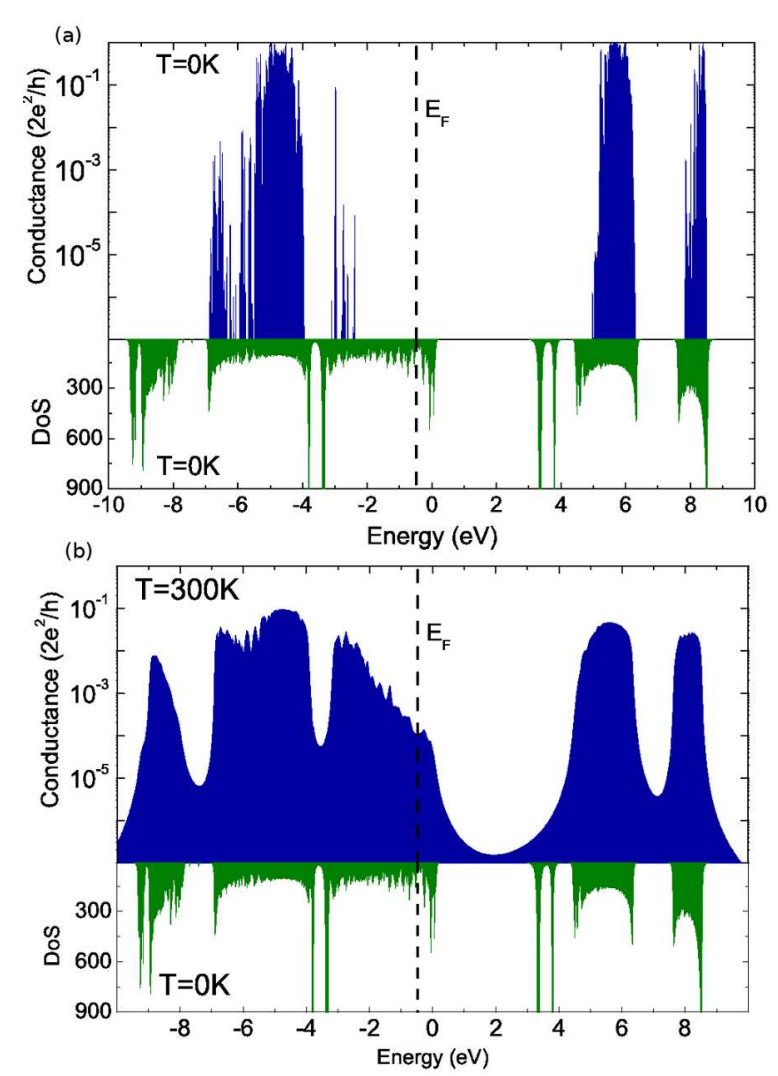

FIG. 3. Conductance for both: (a) $\mathrm{T}=0 \mathrm{~K}$, and (b) $\mathrm{T}=300 \mathrm{~K}$ according to DP model for a 400 rings long PAni- $\mathrm{HCl}$ chain. We also show the $\mathrm{T}=0 \mathrm{~K}$ Density of state for comparison purposes.

of 400 rings [47], we have taken that number in our numerical calculations. However, it should be noted that our results do not depend critically on this parameter. We first calculated the coherent transmission probability as a function of energy for a set of chain configurations drawn from the representative ensemble. Results are in full agreement with the those of Farchioni et al. [14, 15, 48] and evidence the mobility edges induced by correlated disorder in this 1-D system [9]. While there is an appreciable density of states at the Fermi energy, it corresponds to localized states. Indeed, according to Fig. 3 a, the Fermi energy is far away from the extended state region. Fig. 3-b shows the drastic differences in conductance once that decoherent processes are taken into account. Conductance at the Fermi energy now becomes appreciable for any configuration. These results show that metallic transport is possible within a purely $\mathrm{BL}$ model through an environment-assisted transport [45]. Even within a model of perfectly conducting PL islands bridged by BL strands, the calculated chains can be taken as representative of such transport bottleneck. One expects that small differences in quinoid ring concentrations would appear due to natural fluctuations on the oxidation degree previous to doping. In Fig. 4 we show the

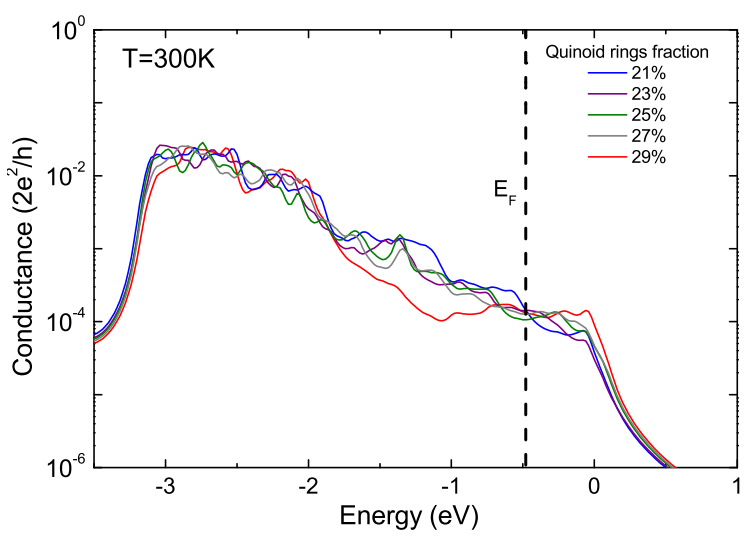

FIG. 4. Fluctuations in total conductance at $\mathrm{T}=300 \mathrm{~K}$ in the main peak around the Fermi energy with the fraction of quinoid rings along the chain.

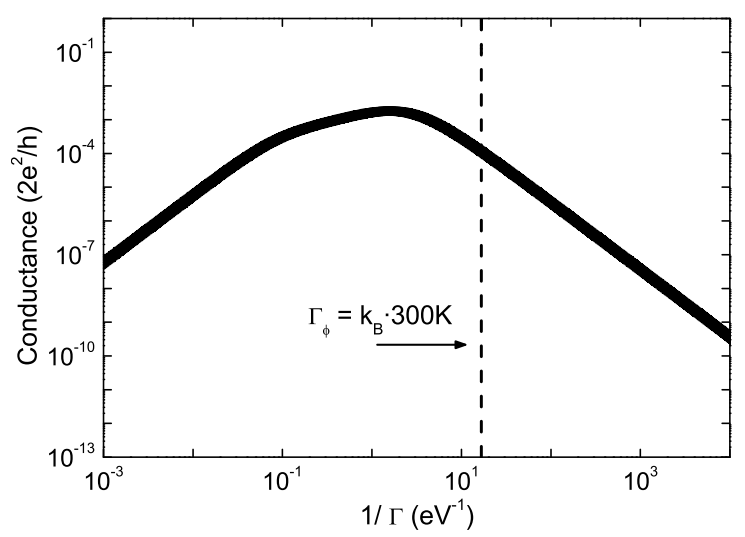

FIG. 5. Conductance for a 400 rings long PAni-HCl chain as a function of $\Gamma_{\phi}$. The value for $\Gamma_{\phi}$ at $T=300 K$ also shown in dash line.

resultant conductance for various quinoid concentrations and found no significant changes in transport.

We also studied the behavior of total conductance as a function of decoherence rate. In accordance with recent works [45, 49], three regimes can be appreciated. Starting from $\mathrm{T}=0 \mathrm{~K}$, as the temperature rises dephasing events become more successful in the destruction of localization caused by coherent interference at the Fermi energy, rising the total conductance of the system. In this regime, transport rate increases as the energy uncertainty associated with temperature is increased. However, there is a $\Gamma_{\phi}$ value for which the conductance is maximal. If the temperature is increased further, the associated energy uncertainty becomes larger than the terms of the system Hamiltonian (characteristic hopping and site energies), and the decoherent process now are able to suppress transport. This is commonly known in the literature as the quantum Zeno effect. In Fig. [5 we show our results for the dependence of total conductance 


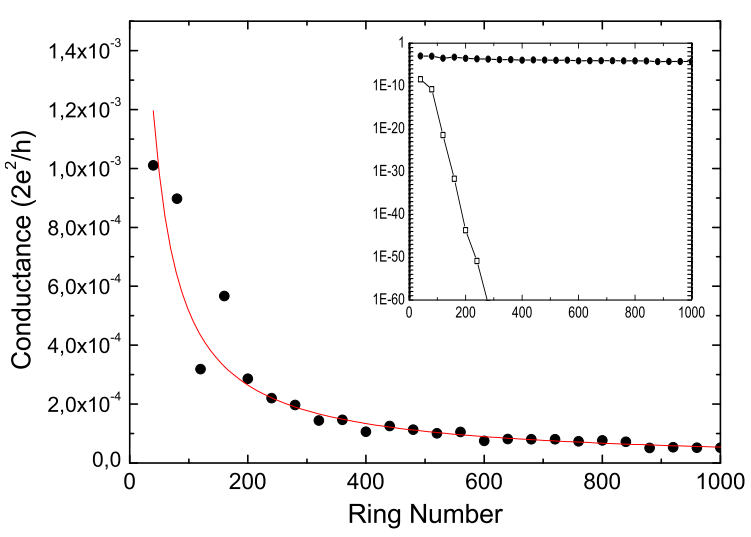

FIG. 6. Total conductance at $\mathrm{T}=300 \mathrm{~K}$ as a function of the chain large. As an inset, it is shown the scale difference between coherent and total conductance.

with $\Gamma_{\phi}$, in which the three regimes above described are clearly seen. The thermal energy, in this case, lies on an area of great influence on the total conductance of this system, and therefore decoherent processes should not be neglected. At room temperatures, the PAni BL is safely placed in the range of thermally assisted transport, and it is clear from the figure that small variations in the exact value of $\Gamma_{\phi}$ do not alter the outcome significantly (note the log-log scale).

We studied the dependence of conductance on the chain length. The results are shown in Fig. 6] With the exception of some fluctuations at short lengths, conductance at $\mathrm{T}=300 \mathrm{~K}$ decreases as the reciprocal of the chain length, as expected for an Ohmic system. The fitting gives $\mathrm{G} /\left(2 e^{2} / h\right)=1 /\left(20,6 N_{R}\right)$, where $N_{R}$ is the number of rings of the chain. The log scale in the inset figure emphasizes the drastic difference between the full conductance and that restricted to coherent tunneling processes. The coherent conductance decays exponentially as expected for a one dimensional disordered system [50]. In our case the localization length is small, $\mathrm{G} /\left(2 e^{2} / h\right)=22 \times 10^{3} e^{-0.53 N_{R}}$, which implies that even for very short disordered polymer chains, transport does not take place unless decoherence processes are involved. Indeed, conductance decays a factor $1 / 3$ for every two rings. Therefore, in a model of islands as that mentioned in the introduction, destruction of localization by decoherence would have a fundamental role. However, our results go further and evidence that even a fully BL PAni would sustain strong electronic transport.

Fig. 7 shows the net current through 50 random chain configurations of bipolaronic PAni. The current was evaluated by using Eq. 13 for symmetric voltages at room temperature. As can be seen, the behavior of different chain configurations is very similar and close to the linear regime. All chain configurations exhibit an appreciable conductance with an average value of $7,7 \times 10^{-9} \Omega^{-1}$. Taking the PAni- $\mathrm{HCl}$ density as $1,329 \mathrm{~g} / \mathrm{cm}^{3}$ [51] we get

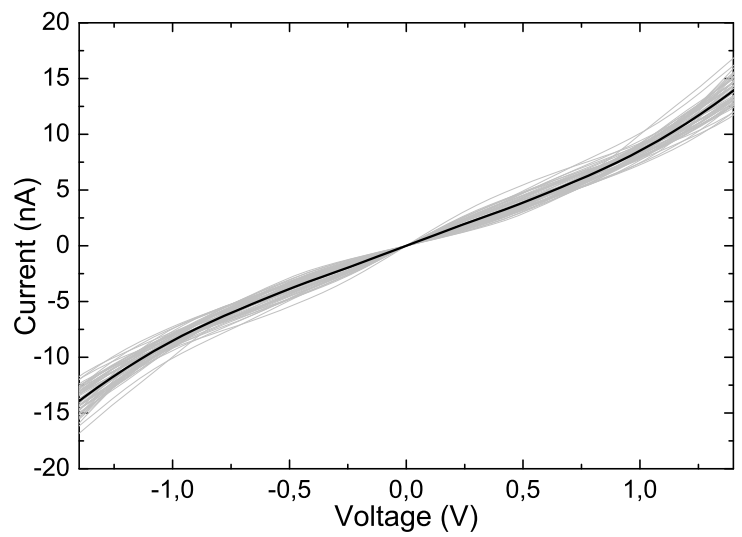

FIG. 7. Average current (black line) and currents for 50 different configurations (shadow lines) are shown.

a conductivity of $\sigma=81 \Omega^{-1} \mathrm{~cm}^{-1}$. This result is slightly higher than the experimental ones, $1 \Omega^{-1} \mathrm{~cm}^{-1} \lesssim \sigma \lesssim$ $20 \Omega^{-1} \mathrm{~cm}^{-1}$ [52] which is reasonable because in the calculation of the conductivity we are considering the conductance of the ideal case of chains directly connected to the leads.

\section{CONCLUDING REMARKS}

In this work we have discussed electron conductance in a doped PAni. We show that the PAni ground state configuration, the $\mathrm{BL}$, has high conductance even in presence of disorder provided that decoherent processes are included. This is done without leaving the convenient $a$ là Landauer approach by using the generalization introduced by D'Amato and Pastawski 32] where an effective transmission accounts for decoherent processes. While our formulation accepts further improvements, it provides an answer from the robust description of Keldysh formalism within a minimal parametrization. Roughly speaking, decoherent processes split each chain into a series of portions whose length is given by the decoherence length $L_{\phi}$ [53]. These define the elemental conductivities from which the sample's Ohmic transport builds on.

For many years, it has been assumed that conduction of polyanilines is inseparably linked to the existence of a polaronic crystalline structure. However, although our main intention is qualitative, we showed that decoherent processes are able to give appreciable metallic conduction in the more entropically favorable bipolaronic lattice. For this system, the uncertainty of energy associated with thermal processes cannot be neglected in the study of conductance, since $k_{B} T$ falls in a region in which the interplay between incoherent and coherent dynamics results in an increased efficiency of electron transport. One might then speculate that only when the thermal energy scale becomes smaller than the Coulomb energy of the localized states, one would actually start to notice 
a qualitative difference with an ideal 1-D metal.

The robustness of the results obtained is evident by noting that they neither depend on variations in the oxidation degree of PAni prior to the doping process, nor on the particular arrangement of quinoid rings along the chain, or on the exact value of the energy uncertainty associated to $\Gamma_{\phi}$. This justifies the fact that good conducting properties do not depend much on the purity of the emeraldine base so that small displacements toward the leucoemeraldine or pernigraniline are acceptable. The evaluation presented in the appendix show that, even when interchain coupling can contribute appreciably to conductivity, the coupling between the $p_{z}$ bonds with torsional degrees of freedom is strong enough to provide almost all the required decoherence. This hypothesis seems consistent with the experiments that show that adding residues that restrict the torsional motion would also diminish the conductivity as compared with the unmodified bipolaronic lattice 54].

We do not attempt to rule out the presence of phase segregation into metallic polaronic islands and "insulating" bipolaronic domains. However, these last strands constitute the bottle-neck where thermal decoherent processes activate the conductivity. Moreover, our results go further ahead and evidence that bipolaronic chains can sustain electronic transport by themselves. In fact, based in our simulations we can estimate bulk conductivity for these chains and arrive to a remarkably good value as compared with experimental data.

\section{ACKNOWLEDGMENTS}

This work is part of an experimental study of PAni based conductors with the support of ANPCyT, CONICET, MiNCyT-Cor and SeCyT-UNC for which the participation of G. A. Monti, P. R. Levstein, R. A. Iglesias and L. J. Gerbino is acknowledged. Discussions with C. Barbero probed also very useful for this work. HMP and RABM also acknowledge the hospitality of BIOTECH at Technical University of Dresden and MPIPKS where these issues where discussed with G. Cunniberti, R. Gutierrez and D. Nozaki.

\section{APPENDIX}

In this section we attempt to elucidate the meaning of $\Gamma_{\phi}$ by presenting two physically meaningful sources of decoherence which must be present in our system: interchain coupling and a simple but general model of electron-phonon interaction. These presentation also allows to compare the similarities and differences between the decoherence rate and the electron-transfer rate in the Marcus-Hush model.

\section{A. Interchain hopping}

We start considering the effect of $V_{X}$, an interchain hopping at site $j$. Any neighboring chain can act as an "environment" for an electron at this site. This is because an electron jumping into a side chain (see Fig. 8) has two options: 1) to escape towards this alternative propagation channel and never return. This is obviously decoherent as it can not interfere any longer with the main pathway [55, 56. 2) to return after having an ergodic walk on the side chain. In this case it is just the excessive amount of interferences and anti-resonances involved that leads to a decoherent description [55]. Each node in the plot corresponds to a multi-chain electronic state. Notice that the interaction structure looks the same as in the local phonon picture discussed in Ref. [25, 26]. Using Eq. [3, we have for self-energy $\Sigma_{j}^{X}$ describing this coupling:

$$
\Sigma_{j}^{X}=\frac{\left|V_{X}\right|^{2}}{\varepsilon-\left(E_{j}-\mathrm{i} \eta\right)-\Sigma_{j}}=\left(\frac{V_{X}}{V_{j, j+1}}\right)^{2} \Sigma_{j},
$$

where $E_{j}, V_{j, j+1}$ are site and hopping strength within the chain. Thus the interchain rate can be expressed as $\Gamma_{\phi}^{X}=$ $\left(\frac{V_{X}}{V_{j, j+1}}\right)^{2} \Gamma_{j}$ where $\Gamma_{j}$ and the imaginary part of total self energies at site $j$. We may evaluate an estimate for the typical $\Gamma_{j}$ by disregarding localization and considering that the side chain is an infinite PAni strand and using the representative values of $\bar{E} \simeq-0.3 \mathrm{eV}$ for and site energy and $\bar{V} \simeq-3.6 \mathrm{eV}$ for intrachain $\pi$ bonds. Thus,

$$
\Gamma_{j}^{X}=\left(\frac{V_{X}}{\bar{V}}\right)^{2} \sqrt{\bar{V}^{2}-\left(\frac{\epsilon-\bar{E}}{2}\right)^{2}} .
$$

We might wonder which range of values would be required from $V_{X}$ to yield an energy uncertainty of the order $\Gamma^{X} \simeq k_{B} T_{R}$ where $k_{B} T_{R}$ stands for room temperature energy. The use of the discussed values yields $V_{X}<\bar{V} / 12$. While we can not ensure that this is the case, in every site of the PAni chain, uncertainty energy associated with interchain coupling is not too far below thermal energy and, therefore, it is it is not negligible. Indeed, there could be sites in which interchain couplings are stronger, and they would contribute substantially to the decay of local states.

\section{B. Torsional Phonon coupling}

Certainly, vibrational degrees of motion are natural sources of decoherence. We analyze them by introducing a simple model for electron-phonon couplings that enables the evaluation of the corresponding contribution to $\Gamma_{\phi}$. From the geometrical inspection of the molecular structure, it is obvious that torsional strains on 


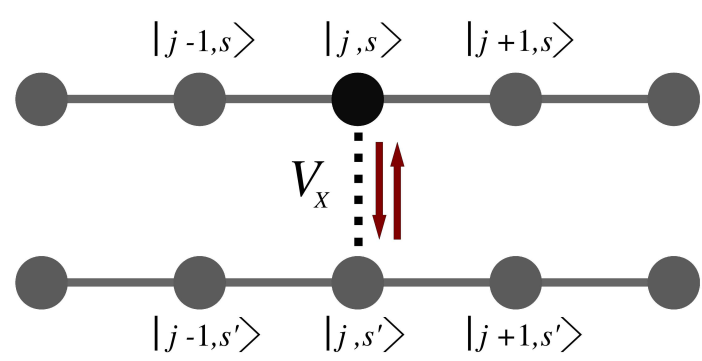

FIG. 8. Interchain hopping at site $j$. States are written in Dirac notation including quantum numbers $s$ which label different PAni chains. This representation illustrates the similarity with Fock-space representation of the electron-phonon system.

benzenoid rings disrupt $\pi$ bonds between $p_{z}$ orbitals of para-Carbons and Nitrogens. Their overlap depends on the angle $\theta$ between the orbital axes. As a result, the corrected hopping energies can be written as $V=V^{0} \cos (\theta) \simeq V^{0}\left(1-\theta^{2} / 2\right)$. The natural frequency $\omega_{\theta}$ of this torsional motion determine the vibrational energy of benzenoid rings. A self consistent description requires that the restoring force $I \omega_{\theta}^{2} \theta$, written in terms of the moment of inertia $I$ of the benzenoid ring, should coincide with the net change in the electronic energy described by the tight-binding model. In this case it yields

$$
V^{0}=I \omega_{\theta}^{2}
$$

leading to $\hbar \omega_{\theta} \simeq 2 \times 10^{-2} \mathrm{eV}<k_{B} T_{R}$.

In terms of the second quantization operators $\hat{b}=$ $\sqrt{I \omega_{\theta} / 2 \hbar}\left(\theta+\mathrm{i} \dot{\theta} / \omega_{\theta}\right)$ and $\hat{b}^{+}=\sqrt{I \omega_{\theta} / 2 \hbar}\left(\theta-i \dot{\theta} / \omega_{\theta \theta}\right)$ we get the perturbation given by the coupling Hamiltonian:

$$
\begin{gathered}
\hat{H}_{e l-p h}=-\frac{1}{4} \hbar \omega_{\theta}\left(\hat{b}^{+}+\hat{b}\right)^{2}\left(\delta_{j^{\prime}, j}+\delta_{j^{\prime}, j-1}\right) \times \\
\sum_{j^{\prime}}\left(\hat{c}_{j^{\prime}}^{+} \hat{c}_{j^{\prime}+1}+\hat{c}_{j^{\prime}+1}^{+} \hat{c}_{j^{\prime}}\right)
\end{gathered}
$$

A Fock-space representation of this interaction Hamiltonian is represented in Fig. 9. Notice the similarities and differences with the representation of the linear electron-phonon interaction discussed in Ref. 25, 26] and the interchain coupling. In the present case, the effect of the perturbation on the state on a local site $j$ can evaluated with the FGR:

$$
\begin{aligned}
\frac{1}{\tau_{j}(\epsilon)}= & \sum_{n} P(n)\left[\frac{2 \pi}{\hbar} \sum_{j^{\prime}, n^{\prime}}\left|\left\langle j, n\left|\hat{H}_{e l-p h}\right| j^{\prime}, n^{\prime}\right\rangle\right|^{2}\right] \times \\
& \delta\left[\left(\epsilon+n \hbar \omega_{\theta}\right)-\left(E_{j^{\prime}}+n^{\prime} \hbar \omega_{\theta}\right)\right]
\end{aligned}
$$

where $|j, n\rangle=\frac{1}{\sqrt{n !}}\left(\hat{b}^{+}\right)^{n} \hat{c}_{j}^{+}|\emptyset\rangle$ here $|\emptyset\rangle$ is the electron and phonon vacuum and $n$ label the number of vibrational quantums whose thermal probability is $P(n)$. In

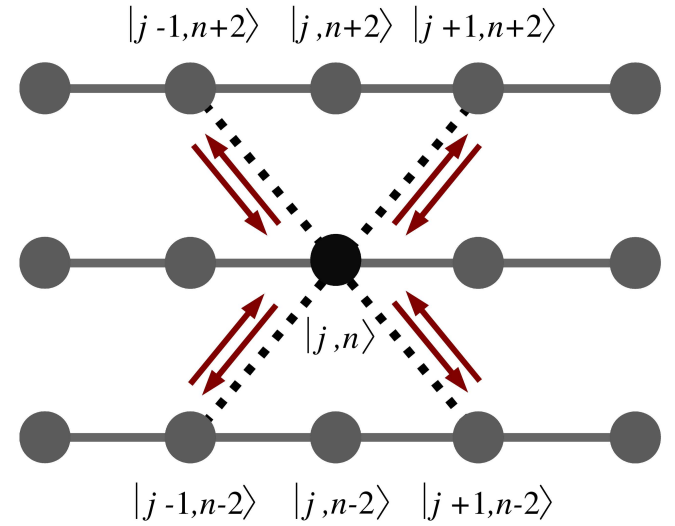

FIG. 9. Fock-space representation of state $|j, n\rangle$ and its surroundings. The middle row represents electronic states with $n$ phonons in the PAni chain. Lower and upper rows represent the same chain but with different numbers of phonons. Black dotted lines are electron-phonon couplings.

the case of interest, we consider electrons at the Fermi level, $E_{F}$. Thus, after energy integration and using the thermal average $\langle\langle n\rangle\rangle \equiv \bar{n}=\sum P(n) n$ for the expectation number of $n$, the decay rate becomes:

$$
\begin{aligned}
\frac{1}{\tau_{j}} & =\frac{\pi}{16 \hbar}\left(\hbar \omega_{\theta}\right)^{2}\left\{\left(\bar{n}^{2}+4 \bar{n}+2\right) N\left(E_{F}-2 \hbar \omega_{\theta}\right)\right. \\
& \left.+2 \bar{n}^{2} N\left(E_{F}+2 \hbar \omega_{\theta}\right)+\left(8 \bar{n}^{2}+8 \bar{n}+1\right) N\left(E_{F}\right)\right\}
\end{aligned}
$$

We must highlight that the quadratic dependence on displacement in the electron-phonon interaction is the responsible for the selection rules that can be appreciated in the last equation. Electrons are allowed to interact with environment only by absorbing or emitting phonon pairs. This is shown in Fig. 9. However, without much loss of generality that $k_{B} T \gg \hbar \omega_{\theta}$, so it is possible to approximate $E_{F} \approx E_{F} \pm 2 \hbar \omega_{\theta}$ and $\bar{n} \approx \frac{k_{B} T}{\hbar \omega_{\theta}}$. As a result,

$$
\frac{1}{\tau_{j}}=\frac{\pi}{8 \hbar}\left(\hbar \omega_{\theta}\right)^{2} N\left(E_{F}\right)\left[12\left(\frac{k_{B} T}{\hbar \omega_{\theta}}\right)^{2}+12\left(\frac{k_{B} T}{\hbar \omega_{\theta}}\right)+3\right]
$$

The evaluation of the corresponding $\Gamma_{\phi}$ becomes trivial in this high-temperature regime,

$$
\Gamma_{\phi}=\frac{\hbar}{2} \frac{1}{\tau_{j}}=\frac{3 \pi}{4} N\left(E_{F}\right)\left(k_{B} T\right)^{2} .
$$

Here, it is crucial to notice that for highly localized states the imaginary self energy results mainly from the decoherent process described above. Thus

$$
\begin{aligned}
N(\epsilon) & \approx \frac{1}{\pi} \frac{\Gamma_{\phi}}{\left(\epsilon-E_{0}\right)^{2}+\Gamma_{\phi}^{2}} \\
& \approx 1 / \pi \Gamma_{\phi} .
\end{aligned}
$$



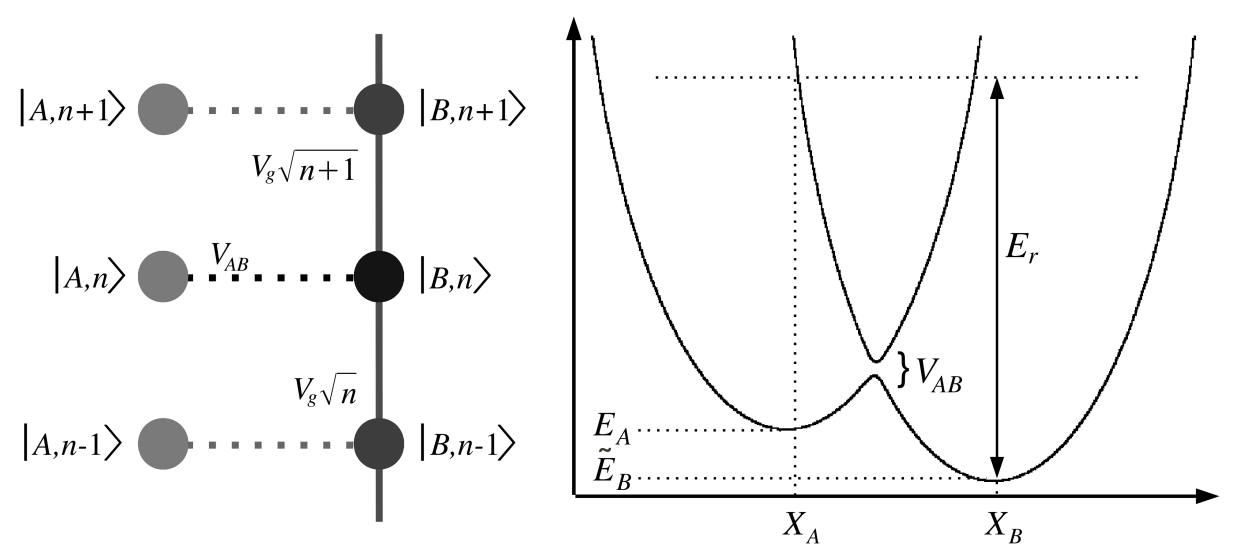

FIG. 10. Fock-space representation of Eq. 30 and its corresponding semiclassical picture in terms of vibronic degree of freedom $X$ as used in the Marcus-Hush model for electron transfer reactions. The potential surface at the right becomes shifted when represented in the polaronic basis allowing to define the reorganization energy $E_{r}$. In this model, the electronic coupling $V_{A B}$ must be small enough to remain perturbative. Left panel is also used to represent the decoherence by electron-phonon coupling when $V_{g} \ll V_{A B}$.

Therefore, from Eq. 24.

$$
\Gamma_{\phi} \sim k_{B} T \text {. }
$$

Thus, any low frequency modes yielding a quadratic dependence of the electronic energy on the displacement, which can be more general than expected, leads to an important consequence: for localized regime, it would tend to provide an energy uncertainty (decoherence) of the order of the thermal energy. A similar behavior remains valid if one relaxes the localization requirement to that of a sharply peaked resonance. A simple example is a sharp resonance in a one dimensional system [57] in which the local density of states could be written:

$$
N(\epsilon)=-\operatorname{Im}\left\{\frac{1}{\pi} \frac{1}{\epsilon-E_{j}+\mathrm{i} \Gamma_{\phi}-\alpha\left(\Delta-\mathrm{i} \Gamma_{b u l k}\right)}\right\},
$$

where $\Gamma_{\text {bulk }}$ represent the escape to the rest of the tightbinding chain and $\Delta$ gives the energy shift due the presence of the other sites. For $\epsilon$ within the band edges, this equation results:

$$
N(\epsilon)=\frac{1}{\pi \Gamma} \frac{1+2 \alpha \Gamma_{b u l k} / \Gamma}{\left(\frac{\epsilon-E_{j}-2 \alpha \Delta}{\Gamma}\right)^{2}+\left(1+2 \alpha \Gamma_{b u l k} / \Gamma\right)^{2}},
$$

which in the limit of large $\Gamma$ compared with $\alpha \Gamma_{b u l k}$, gives $N(\epsilon) \approx 1 / \pi \Gamma$. This limit is achieved at room temperature whenever $\alpha \ll 1$.

\section{Comparison to Marcus-Hush theory}

In the previous section we deemed with the physically relevant situation of a quadratic interaction with the vibrational coordinate. This is not conceptually different with the standard linear electron-phonon coupling used to describe the Franck-Condon effect [58] and the electron-transfer process [59]. All these physical processes are contained in a simple Hamiltonian

$$
\begin{gathered}
\hat{H}=\sum_{j=A, B} E_{j} \hat{c}_{j}^{+} \hat{c}_{j}+\hbar \omega_{0}\left(\hat{b}^{+} \hat{b}+\frac{1}{2}\right)-V_{g}\left(\hat{b}^{+}+\hat{b}\right) \hat{c}_{B}^{+} \hat{c}_{B} \\
+V_{A B}\left(\hat{c}_{A}^{+} \hat{c}_{B}+\hat{c}_{B}^{+} \hat{c}_{A}\right),
\end{gathered}
$$

whose interactions in the Fock space are represented in Fig. 10. The electron transfer problem is best represented resorting to the polaronic transformation which would diagonalize the Hamiltonian but for the tunneling described by $V_{A B}$. The essence of an estimation of the electron transfer rate is a FGR evaluation of the tunneling between the electronic states $\mathrm{A}$ and $\mathrm{B}$ in the regime of weak coupling non-adiabatic limit $\hbar \omega_{0} \ll$ $k_{B} T,\left|V_{A B}\right| \ll\left|V_{g}\right|$.

$$
k_{A \longrightarrow B}=\frac{1}{\tau_{A \longrightarrow B}}=\frac{2 \pi}{\hbar}\left|V_{A B}\right|^{2}[F(\Delta E)]
$$

where $\Delta E=E_{A}-\tilde{E}_{B}, \tilde{E}_{B}=E_{B}-V_{g}^{2} / \hbar \omega_{0}$ and $F(\Delta E)$ is a density of directly connected states denominated Franck-Condon factor. Thus it satisfies

$$
\int_{-\infty}^{\infty} F(\Delta E) \mathrm{d} \Delta E=1
$$

$F(\Delta E)$ is estimated resorting to a thermal average and following the Marcus original treatment which interprets the transition probability according to a Landau-Zener formula. Thus

$$
F(\Delta E)=\frac{1}{\sqrt{4 \pi E_{r} k_{B} T}} \exp \left[-\frac{\left(\Delta E-E_{r}\right)^{2}}{4 E_{r} k_{B} T}\right]
$$


where the reorganization energy $E_{r}$ is indicated in the plot.

In contrast to this treatment, in a decoherence problem one focus on estimating how the electron-phonon interaction degrades the standard coherent Rabi oscillation [60]. This describes an electron jumping forth and back between states B and A and atenuates within a decoherence time $\tau_{\phi}$. Similarly to Eq. (3.14) of Ref. [33] a FGR evaluation gives:

$$
\begin{aligned}
\frac{1}{\tau_{\phi}} & =\frac{2 \pi}{\hbar}\left|V_{g}\right|^{2}\left\langle\left\langle(n+1) N\left(E_{B}+\hbar \omega_{0}\right)+n N\left(E_{B}-\hbar \omega_{0}\right)\right\rangle\right\rangle \\
& \simeq \frac{4 \pi}{\hbar}\left|V_{g}\right|^{2}\left[\frac{k_{B} T}{\hbar \omega_{0}} N\left(E_{B}\right)\right] .
\end{aligned}
$$

where $\langle\langle\rangle\rangle$ stands for thermal average. The approximation involves a high temperature limit and again, the square brackets indicate a density of directly connected states. As in previous section, the assumption that phonon induced electron energy uncertainty leads to the self-consistent condition of Eq. 26.

$$
\Gamma_{\phi}=\frac{\hbar}{2 \tau_{B}}=\left|V_{g}\right| \sqrt{\frac{2 \pi k_{B} T}{\hbar \omega_{0}}},
$$

which is valid provided that $\hbar \omega_{0} \ll k_{B} T,\left|V_{g}\right| \ll V_{A B}$. Notice that in an ab-intio parametrization of the tightbinding Hamiltonian, the coupling constant results from evaluating the dependence of the parameter on the appropriate generalized coordinate, e.g. $V_{g}=\partial E_{B} / \partial \theta$.

Thus, in both problems, electron transfer in presence of a some reorganization energy and electron transport with decoherence from a phonon bath, the Hamiltonian is the same. However, since the calculated observables are different, the term used as perturbation in the FGR differ. In the first case the perturbation is the electron jump $V_{A B}$, while in the decoherent situation the perturbation is the electron-phonon coupling constant $V_{g}$.
[1] A. J. Heeger, Rev. Mod. Phys. 73, 681 (2001).

[2] K. Fehse, G. Schwartz, K. Walzer, and K. Leo, J. Appl. Phys. 101, 124509 (2007).

[3] E. M. Conwell, Physics Today, 38(6) 46 (1985).

[4] W.P. Su, J.R. Schrieffer and A.J. Heeger, Phys. Rev. Lett. 42, 1698 (1979).

[5] A. J. Heeger, J. Phys. Chem. B 105, 8475 (2001).

[6] P. W. Anderson, Rev. Mod. Phys. 50, 191 (1978); A. Mac Kinnon and B. Kramer, Rep. Prog. Phys. 561469 (1993).

[7] D. S. Galvão, D. A. dos Santos, B. Laks, C. P. de Melo, and M. J. Caldas, Phys. Rev. Lett. 63, 786 (1989).

[8] H.-L. Wu and P. Phillips, Phys. Rev. Lett. 66, 1366 (1991).

[9] D. H. Dunlap, H.-L. Wu, and P. W. Phillips, Phys. Rev. Lett. 65, 88 (1990).

[10] P. Phillips, Advanced Solid State Physics, Westview (2003).

[11] Y. Imry and R. Landauer, Rev. Mod. Phys. 71, S306 (1999).

[12] C. Joachim, J. K. Gimzewski and A. Aviram, Nature 408, 541 (2000); J. R. Heath and M. A. Ratner, Phys. Today 56 (5) 43 (2003).

[13] P.A. Schulz, D. S. Galvão and M. J. Caldas, Phys. Rev. B 44, 6073 (1991).

[14] R. Farchioni, P. Vignolo, and G. Grosso, Phys. Rev. B 60, 15705 (1999).

[15] P. Vignolo, R. Farchioni, and G. Grosso, Phys. Stat. Sol. B 223, 853 (2001).

[16] N. S. Sariciftci, A. J. Heeger and Y. Cao, Phys. Rev. B 49, 5988 (1994).

[17] C. Cavazzoni, R. Colle, R. Farchioni, and G. Grosso, Phys. Rev. B 66, 165110 (2002).

[18] A. Varela-Álvarez and J. A. Sordo, J. Chem. Phys. 128, 174706 (2008).

[19] J. M. Ginder, A. F. Richter, A. G. MacDiarmid and A. J. Epstein, Solid State Commun. 63, 97 (1987); A. J. Epstein, J. M. Ginder, F. Zuo, R. W. Bingelow, H. S. Woo, D. B. Tanner, A. F. Richter, W. S. Huang and A.
G. MacDiarmid, Synth. Met. 18, 303 (1987).

[20] A. Petr, A. Neudeck and L. Dunsch, Chem. Phys. Lett. 401, 130 (2005).

[21] S. Stafström, J. L. Brédas, A. J. Epstein, H. S. Woo, D. B. Tanner, W. S. Huang and A. G. MacDiarmid, Phys. Rev. Lett. 59, 1464 (1987)

[22] V. N. Prigodin and A. J. Epstein, Synth. Met. 125, 43 (2002); V. N. Prigodin and A. J. Epstein, Physica B. 338, 310 (2003).

[23] H. C. F. Martens and H. B. Brom, Phys. Rev. B 70, 241201(R) (2004).

[24] H. C. F. Martens, Phys. Rev. Lett. 96, 076603 (2006).

[25] H. M. Pastawski, L. E. F. Foa Torres and E. Medina, J. Chem. Phys. 281, 257 (2002).

[26] L. E. F. FoaTorres, H. M. Pastawski and S. S. Makler, Phys. Rev. B 64193304 (2001).

[27] E.P. Danieli, G.A. Alvarez, P.R. Levstein, and H.M. Pastawski, Solid State Commun. 141, 422 (2007).

[28] H. M. Pastawski and G. Usaj, Phys. Rev. B 57, 5017 (1998).

[29] S. Stafström. Phys. Rev. B. 51, 4137 (1995).

[30] F. Milde, R.A. Römer, M. Schreiber and B. Uski, Eur. Phys. J. B, 15, 685 (2000).

[31] P. R. Levstein, H. M. Pastawski, and J. L. D'Amato, J. Phys.: Condens. Matter 2, 1781 (1990).

[32] J. L. D'Amato and H. M. Pastawski, Phys. Rev. B 41, 7411 (1990).

[33] H. M. Pastawski, Phys. Rev. B 46, 4053 (1992).

[34] K. Maschke and M. Schreiber, Phys. Rev. B 49, 2295 (1994).

[35] R. Hey, F. Gagel, M. Schreiber and K. Maschke, Phys. Rev. B 55, 4231 (1997).

[36] R. Hey and M. Schreiber, Phys. Rev. B 56, 1854 (1997).

[37] N. A. Zimbovskaya, J. Chem. Phys. 129, 114705 (2008).

[38] R. Gutiérrez, S. Mandal, and G. Cuniberti, Phys. Rev. B 71, 235116 (2005)

[39] D. Nozaki, Y. Girard, and K. Yoshizawa, J. Chem. Phys. C 112, 17408 (2008).

[40] H. M. Pastawski, Phys. Rev. B 44, 6329 (1991). 
[41] D. M. Basko, I. L. Aleiner, and B. L. Altshuler, Ann. Phys. N.Y. 321, 1126 (2006).

[42] R. A. Marcus, Rev. Mod. Phys. 65, 599 (1993).

[43] H. M. Pastawski and E. Medina, Rev. Mex. Fis. Vol. 47 (S1) 1 (2001).

[44] S. L. Sondhi, S. M. Girvin, J. P. Carini and D. Shahar, Rev. Mod. Phys. 69, 315 (1997).

[45] P. Rebentrost, M. Mohseni, I. Kassal, S. Lloyd and A. Aspuru-Guzik, New J. Phys. 11, 033003 (2009); contains an estimation of $\Gamma_{\phi} \simeq k_{B} T$.

[46] X. Q. Li and Y. Yan, Appl. Phys. Lett. 79, 2190 (2001); J. Chem. Phys. 115, 4169 (2001).

[47] H. S. Kolla, S. P. Surwade, X. Zhang, A. G. MacDiarmid and S. K. Manohar, J. Am. Chem. Soc. 127, 16770 (2005).

[48] R. Farchioni, G. Grosso, and G. P. Parravicini, Phy. Rev. B 53, 4294 (1996).

[49] M. Mohseni, P. Rebentrost, S. Lloyd and A. AspuruGuzik, J. Chem. Phys. 129, 174106 (2008); for previous results see Ref. [32].

[50] A. Mac Kinnon and B. Kramer, Phys. Rev. Lett. 47, 1546 (1981); H. M.Pastawski, C. M. Slutzky and J. F. Weisz, Phys. Rev. B 32, 3642 (1985).

[51] J. Stejskal and R. G. Gilbert, Pure Appl. Chem., 74(5) 857 (2002).

[52] X.-R. Zeng, K.-C. Gong, K.-N. Weng, W.-S. Xiao, W.-H. Gan and T.-M. Ko, Chem. Phys. Lett. 280, 469 (1997); C.-T. Kuo and C-H Chen, Synth. Met. 99, 163 (1999);
J. H. Huang, Phys. Rev. B 58, 12 (1998); Y. Longa, Z. Chena, N. Wanga, Z. Zhang and M. Wan, Physika B 325, 208 (2003).

[53] H. M. Pastawski, J. F. Weisz, and E. A. Albanesi, Phys. Rev. B 52, 10665 (1995).

[54] C. -C. Han, S. -P. Hong, K. -F. Yang, M. -Y. Bai, C. -H. Lu, and C. -S. Huang, Macromolecules 34, 587 (2001); C. -C. Han, C. -H. Lu, S. -P. Hong, and K. -F. Yang, Macromolecules 36, 7908 (2003); H. J. Salavagione, D. F. Acevedo, M. C. Miras, A. J. Motheo, C. A. Barbero, J. Polym. Sci., Polym. Chem. 42, 5587 (2004).

[55] L. E. F. Foa Torres, H. M. Pastawski and E. Medina, Europhys. Lett. 73164 (2006).

[56] J. Bonča and S. A. Trugman, Phys. Rev. Lett. 75, 2566 (1995); J. Bonča, S. A. Trugman, and I. Batistić, Phys. Rev. B 60, 1633 (1999); E. V. Anda, S. S. Makler, H.M. Pastawski and R. G. Barrera., Braz. J. Phys. 24, 330 (1994).

[57] E. Rufeil-Fiori, C. M. Sanchez, F. Y. Oliva, H. M. Pastawski, and P. R. Levstein, Phys. Rev. A 79, 032324 (2009).

[58] M. P. Marder, Condensed Matter Physics, (Wiley, New York, 2000) p.628.

[59] A. Nitzan, Chemical Dynamics in Condensed Phases. Relaxation, Transfer and Reactions in Condensed Molecular Systems, (Oxford University Press, New York, 2007), see Chap. 16.

[60] H. M. Pastawski, Physica B 398, 278 (2007). 